\title{
Magnetic Resonance Image in the diagnosis and evaluation of extra-prostatic extension and involvement of seminal vesicles of prostate cancer: a systematic review of literature and meta-analysis
}

Rogério Cardoso da Silva, André Deeke Sasse, Wagner Eduardo Matheus, Ubirajara Ferreira

Department of Uro-oncology (RCS, WEM, UF) and Center of Oncologic Evidences (ADS), Universidade Estadual de Campinas, UNICAMP, Campinas, Brazil

\section{ABSTRACT}

Objective: Systematic review of literature and meta-analysis to evaluate the results of magnetic resonance image $1.5 \mathrm{~T}$ with endorectal coil in the diagnosis and evaluation of extra-prostatic extension and involvement of seminal vesicles of prostate cancer, compared to the histopathological results of the radical prostatectomy specimen.

Materials and Methods: It was conducted a systematic review of literature and meta-analyses of all studies data published after 2008. In those studies, the patients with prostate cancer with indication to radical prostatectomy were submitted to magnetic resonance image (MRI) at pre-operatory period and the results were compared to those of histopathological studies after the surgery. The selected terms for research included prostate cancer, magnetic resonance, radical prostatectomy, and prostate cancer diagnosis, in the databases EMBASE, LILACS, PUBMED/MEDLINE and Cochrane Library. The data were collected using a specific qualitative instrument and the meta-analysis data were presented in the forest plot graphics, homogeneity test and sROC curves and funnel plot. Results: A total of seven studies were included, with a total of 603 patients. Among these studies, six evaluated the value of MRI for the detection of prostate cancer, and the median sensitivity of meta-analysis was 0.6 and specificity 0.58 , but with heterogeneity among the studies. Three studies evaluated extra-prostatic extension with a median sensitivity of 0.49 , specificity 0.82 and heterogeneity only for sensitivity. Three studies evaluated invasion of seminal vesicles, with median sensitivity of 0.45 and specificity 0.96 , with heterogeneity in both analysis.

Conclusion: Magnetic resonance of $1.5 \mathrm{~T}$ with endocoil showed low values of sensitivity and specificity for the diagnosis and staging of prostate cancer. The reviewed studies showed a significant heterogeneity among them. The best observed result was MRI specificity for invasion of seminal vesicles. More studies are necessary to evaluate new techniques and parameters before recommending the routine use of MRI in clinical practice.

\section{ARTICLE INFO}

\section{Key words:}

Prostatic Neoplasms;

Magnetic Resonance

Spectroscopy; Diagnosis

Int Braz J Urol. 2013; 39: 155-66

Submitted for publication:

December 10, 2012

Accepted after revision:

April 02, 2013

\section{INTRODUCTION}

Prostate cancer (PC) is the most frequent malign tumor among men older than 50 years and presents a tendency of increase in the next decades due to the rise of life expectancy (1).

Although the natural history of the disease is not completed understood, several prognostic 
factors influence the evolution of PC. Among these, some are very important: histological grade, PSA level, tumor volume and extension of the disease $(2,3)$.

There is a lack of consensus in clinical practice regarding diagnostic, staging and treatment techniques that may lead to excessive and unnecessary image exams with increasing costs, waste of time and excessive exposure to ionizing radiation (depending upon the used method) $(4,5)$.

Magnetic resonance image (MRI) with endorectal coil is the most accepted diagnostic method for the evaluation of the prostate tumor, with the advantage of not exposure to ionizing radiation. But there are still several doubts regarding its usefulness and real applicability.

The objective of the present study was the systematic review (SR) of studies that used MRI with conventional 1.5T images in the diagnosis and staging of PC compared to the results of histopathological studies of the radical prostatectomies (RP).

It was performed a systematic meta-analyses, including sensitivity and specificity, comparing the results regarding diagnosis, evaluation of extra-prostatic extension (EPE) and invasion of seminal vesicles (ISV).

\section{MATERIALS AND METHODS}

We selected studies in which MRI was used for PC diagnosis, extension of extra-prostatic disease evaluation and involvement of seminal vesicles compared to histopathological studies of RP.

We included clinical trials with the following inclusion criteria: transversal studies published after 2008 and all patients presenting PC at biopsy with indication of RP without previous treatment. All patients should have been submitted to MRI previous to surgery with conventional $1.5 \mathrm{~T}$ images.

The following databases were reviewed using the terms prostate cancer, magnetic resonance, radical prostatectomy and diagnosis: PUBMED, MEDLINE, EMBASE, LILACS and Cochrane Library. The following terms were used for PUBMED: (("prostaticneoplasms "[MeSHTerms] OR ("prostatic"[AllFields] AND
"neoplasms"[AllFields]) OR "prostaticneoplasms "[AllFields]) AND ("prostatectomy”[MeSHTerms] OR “prostatectomy”[AllFields]) AND ("magn eticresonancespectroscopy"[MeSHTerms] OR ("magnetic"[AllFields] AND "resonance"[AllFields] AND "spectroscopy"[AllFields]) OR "magn eticresonancespectroscopy"[AllFields] OR ("magnetic"[AllFields] AND "resonance"[AllFields]) OR "magneticresonance"[AllFields]))AND Diagnosis/Narrow[filter]. The same search strategy was adapted for the remaining databases. There was no restriction of the published language. Two independent researchers selected the papers and collected the relevant data. For this purpose, it was used a data collection protocol, depicting the studies characteristics and the collected results. In order to solve any possible disagreements between the researchers, a third one was convened.

To evaluate the individual quality of the studies it was used the question form QUADAS (Quality Assessment of Diagnostic Accuracy Studies). The questions included the main sources of bias and each question should have been answered with "yes", "no", "not clear". The greatest the number of negative or not clear answers, the worst was the quality of the study (6).

\section{Meta-analysis and presentation of results}

We used the following software for the meta-analysis: RevMan version 6 (Cochrane) and Meta DiSc 1.4. The presentation of the results used four statistical tools: forest plot, homogeneity test, sROC curve and funnel plot.

1. Forest plot: it was used a statistical combination of sensitivity and specificity of each study, obtaining a median integrated value (pooling) (7).

2. Homogeneity tests: it was used the chi-square test (Q), and the inconsistency index (I-squared, $\mathrm{I}^{2}$ ) to estimate the heterogeneity of the individual studies. $\mathrm{P}<0,05$ suggested heterogeneity. I-squared $\left(\mathrm{I}^{2}\right)$ describes the percentage of total variation among the studies and $\mathrm{I}^{2}>50 \%$ suggested significant heterogeneity among data (8).

3. sROC curve: summarizes and combines rates of true positive and false positive diagnosis 
of the different studies. AUC (area under the curve) summarizes the quality of the curve, representing an index of accuracy of the test. A test with low performance has AUC $<0.5$ and a satisfactory one AUC $>0.70$. Q* evaluates the point at the sROC curve where sensitivity and specificity are equal, being equivalent to the point of symmetry of the sROC curve. If $Q^{*}$ is $\leq 0.5$ the test is not worth for the studied evaluation and as much $\mathrm{Q}^{*}$ approaches 1.0 the best performance has the test (9).

4. Funnel plot: the published bias can be detected by the use of the funnel plot chart, in which the size of the sample is the strongest correlate of the bias of the publication. In the absence of bias, the data have a more funneled symmetric distribution. The presence of a not symmetric funneled distribution indicates the presence of bias of publication $(10,11)$. Cochran- $Q$ with $P<0.05$ suggests the presence of heterogeneity beyond what expected singly. I-squared $\left(\mathrm{I}^{2}\right)$ describes the percentage of variability effect due to heterogeneity and not due to random distribution. $\mathrm{I}^{2}>50 \%$ disclose heterogeneity. $\mathrm{T}^{2}$ estimates variability among studies, and $\mathrm{T}^{2}>1$ suggests heterogeneity $(10,11)$.

\section{RESULTS}

\section{General Aspects}

Using the search terms, 234 summaries were selected from the online databases. After careful evaluation of the summaries, 27 articles were selected for careful review, and after that, 20 were excluded. The main causes of exclusion of the 20 articles included: 6 with any previous treatment prior do RP, 4 without correct identification of the use of the endocoil, 5 without sufficient data for the calculus of sensitivity and specificity. Seven studies were selected, 6 in English and 1 in German (Figure-1).

All seven selected studies were transversal (without calculus of the size sample), unicentric (one only institution involved) and summed 603 patients (Table-1).

Most studies were considered with good consistency after the use of QUADAS question form, sin-
Figure 1 - Organogram of the selected, examined and included studies of SR.

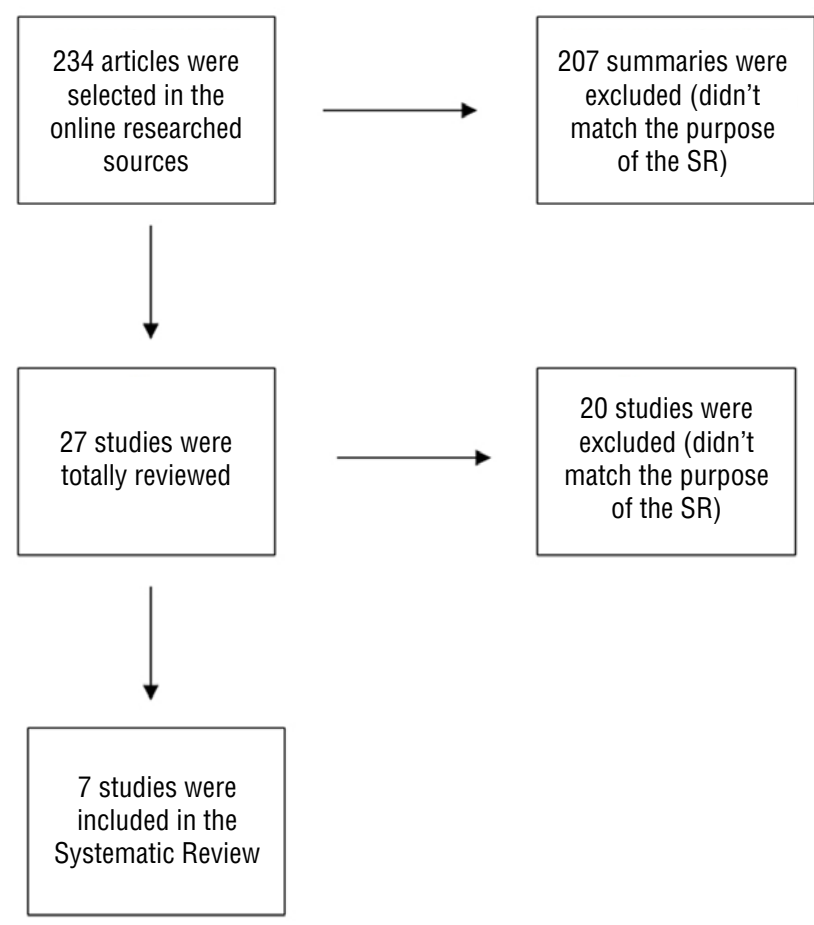

ce there were mainly "yes" answers after methodological evaluation (greater than 50\% of the answers). The question "if the results of the gold-standard test (histopathological study of RP) were interpreted without the knowledge of the test being evaluated (pre-operatory MRI)" had not a satisfactory answer.

\section{Analysis of the diagnosis of PC}

Six of the seven selected articles studied the capacity of MRI with endocoil and 1.5T conventional images to diagnose PC. The mean sensitivity of this meta-analysis was 0.6 and specificity 0.58 (the data were obtained from the florest plot).

The homogeneity test for sensibility and specificity showed $\mathrm{Q}=36.49(\mathrm{P}=0.000), \mathrm{I}^{2}=$ $86.3 \%$ and $\mathrm{Q}=24.89(\mathrm{P}=0.0001), \mathrm{I}^{2}=79.9 \%$, respectively (Figures 2a and 2b). sROC curve showed AUC $=0.7090$ and $Q^{*}=0.6601$ (Figure-3).

Funnel plot showed Cochran- $\mathrm{Q}=26.80(\mathrm{P}$ $=0.0001), \mathrm{I}^{2}=81.3 \%$ and $\mathrm{T}^{2}=1.1861$ (Figure-4) There was a tendency of displacement of the graphic symmetry to the right demonstrating heterogeneity in the statistical parameters of the $Q$ test of Cochran, $\mathrm{I}^{2}$ and $\mathrm{T}^{2}$. 
Table 1 - Summarized data of the studies used in the SR.

\begin{tabular}{lccccccc}
\hline Author & Year & Country & TR & No Patients & Age (Years) & PSA(ng/dl) & RP \\
\hline Chabanova et al (15) & 2010 & Denmark & P & 43 & $64.1(51-74)$ & $10.2(1,3-28)$ & Open \\
Kim et al (18) & 2010 & Canada & R & 32 & $59.1(52.4-65.8)$ & $\#$ & Open \\
Brajtbord et al (19) & 2011 & USA & R & 179 & 59.3 & 6.6 & RALP \\
Janane et al (14) & 2011 & Morocco & NC & 190 & $62.9(50-73)$ & $10.8(2-18,5)$ & Open \\
Jeong et al (12) & 2010 & South Korea & R & 88 & $66(41-76)$ & $5.3(1.5-9.8)$ & Open \\
Yoshizako et al (13) & 2008 & Japan & R & 23 & $65(52-76)$ & NM & Open \\
Beyersdorff et al (16) & 2011 & Germany & P & 48 & $63.43(49-71)$ & $8.17(2.7-31.4)$ & Open \\
\hline
\end{tabular}

$\#<10 \Rightarrow 2$ patients; $10-20 \Rightarrow 5$ patients; $>20 \Rightarrow 5$ patients; RALP; Robotic assisted

TR: Temporal relation; P: prospective, R: retrospective, NC: not clear; RP: Radical prostatectomy; NM: Not mentioned

Figure 2 - Forest plot of sensitivity and specificity regarding the diagnosis of PC.

A

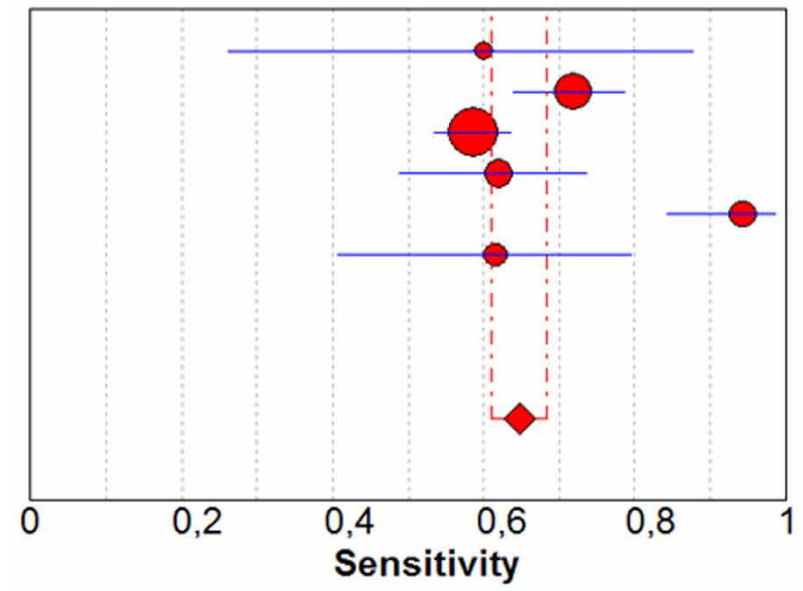

Beyersdorff 2011

Chabanova 2010 Janane 2011

Jeong 2010

Kim 2010

Yoshizako 2008

\section{Sensitivity $(95 \% \mathrm{Cl})$}

$0,60 \quad(0,26-0,88)$

$0,72 \quad(0,64-0,79)$

$0,59 \quad(0,53-0,64)$

$0,62 \quad(0,49-0,74)$

$0,94 \quad(0,84-0,99)$

$0,62 \quad(0,41-0,80)$

Pooled Sensitivity $=0,65(0,61$ to 0,68$)$

Chi-square $=36,49 ; d f=5(p=0,0000)$

Inconsistency (I-square) $=86,3 \%$

B

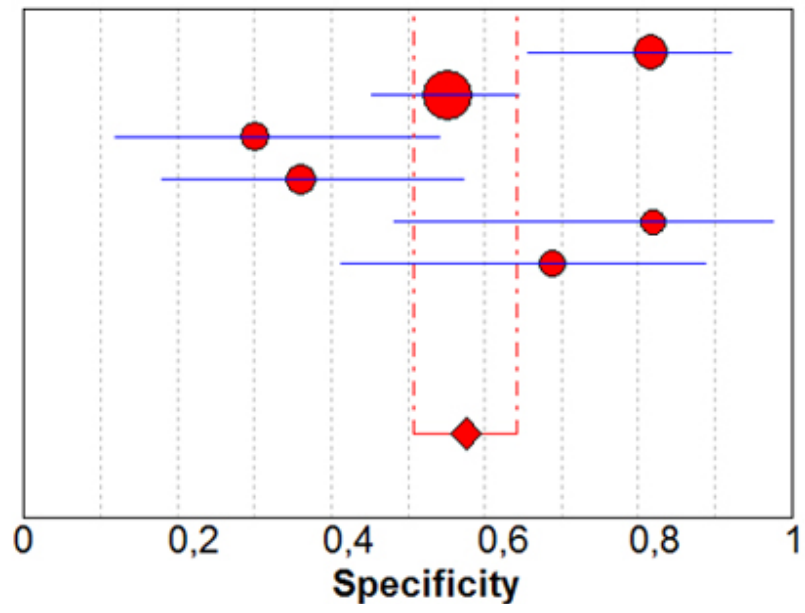

Beyersdorff 2011

Chabanova 2010

Janane 2011

Jeong 2010

Kim 2010

Yoshizako 2008
Specificity $(95 \% \mathrm{Cl})$

$0,82 \quad(0,66-0,92)$

$0,55 \quad(0,45-0,65)$

$0,30 \quad(0,12-0,54)$

$0,36 \quad(0,18-0,57)$

$0,82 \quad(0,48-0,98)$

$0,69 \quad(0,41-0,89)$

Pooled Specificity $=0,58(0,51$ to 0,64$)$

Chi-square $=24,89 ; d f=5(p=0,0001)$

Inconsistency (I-square) $=79,9 \%$ 
Figure 3 - sROC curve depicting the representation of sensitivity versus 1-specificity of the diagnosis of PC.

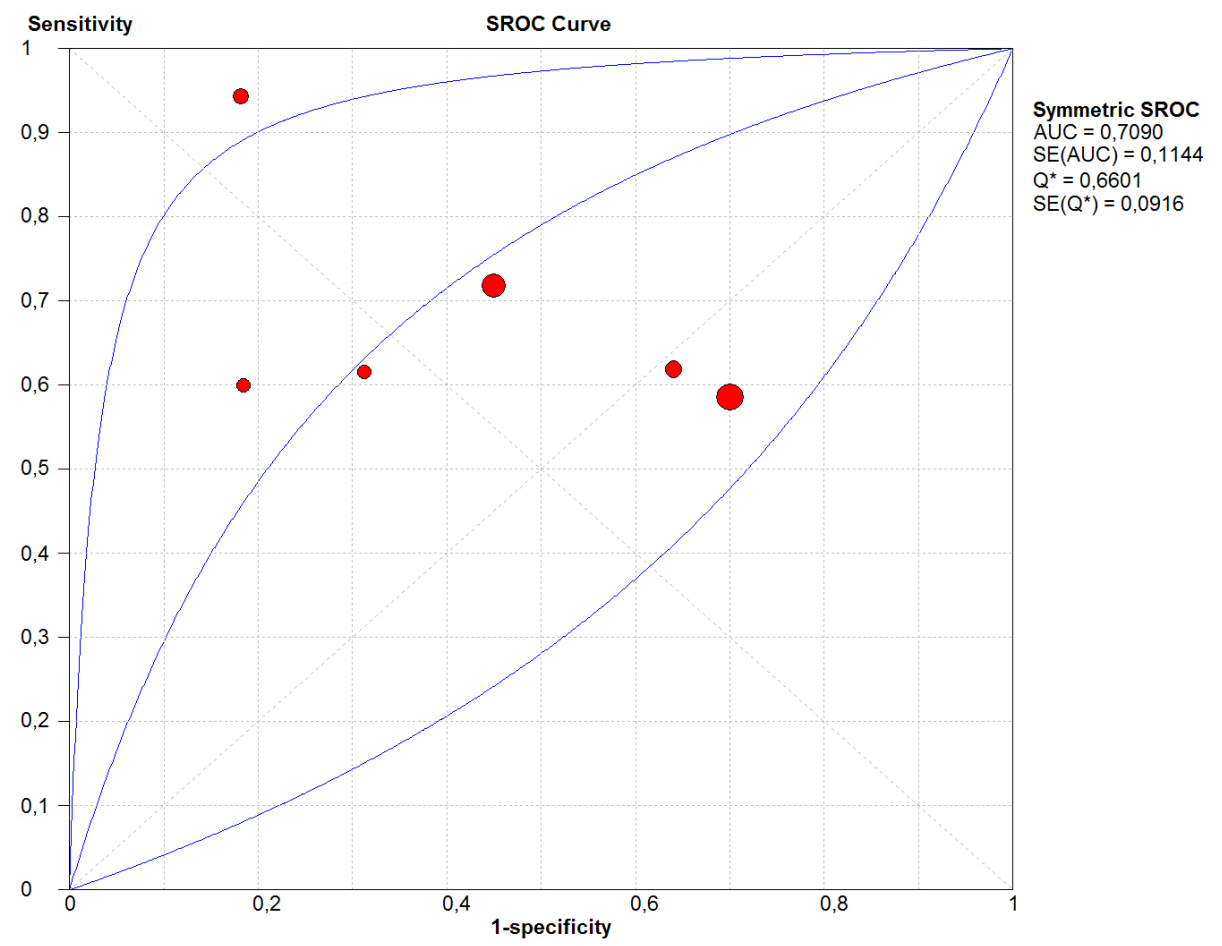

Figure 4 - Funnel plot of the studies regarding the diagnosis of PC.

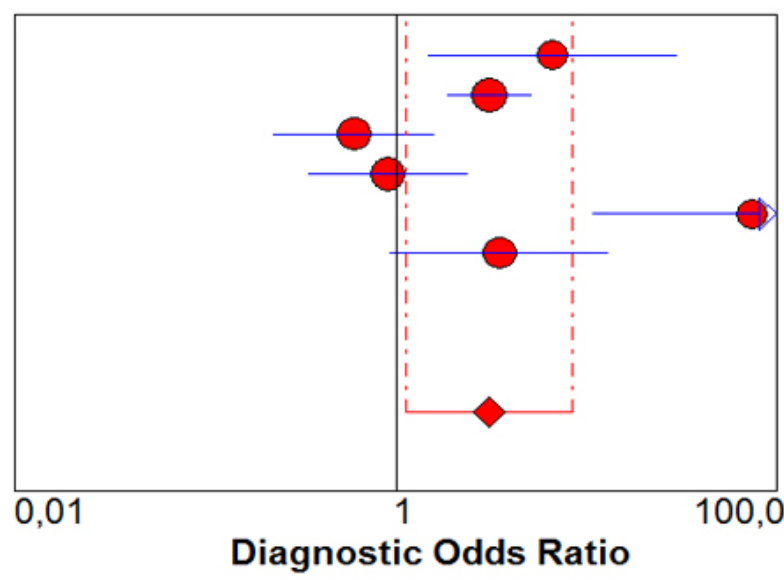

Beyersdorff 2011

Chabanova 2010

Janane 2011

Jeong 2010

Kim 2010

Yoshizako 2008
Diagnostic OR $(95 \% \mathrm{Cl})$

$6,64 \quad(1,47-30,00)$

$3,12(1,86-5,24)$

$0,61 \quad(0,23-1,61)$

$0,91 \quad(0,35-2,39)$

$75,00 \quad(10,94-514,04)$

$3,52 \quad(0,94-13,17)$

Random Effects Model

Pooled Diagnostic Odds Ratio $=3,10(1,14$ to 8,44$)$

Cochran- $Q=26,80 ; d f=5(p=0,0001)$

Inconsistency (I-square) $=81,3 \%$

Tau-squared $=1,1861$ 


\section{Analysis of EPE}

Three of the seven studies evaluated the capacity of MRI with conventional 1.5T images to detect EPE, with a median sensibility of 0.49 and specificity of 0.82 .

The homogeneity test for sensitivity and specificity showed $\mathrm{Q}=16.28(\mathrm{P}=0.0006), \mathrm{I}^{2}=87.7 \%$ and $\mathrm{Q}=1.09(\mathrm{P}=0.5799), \mathrm{I}^{2}=0.0 \%$, respectively $(\mathrm{Fi}-$ gures $5 \mathrm{a}$ and $5 \mathrm{~b})$. sROC curve showed AUC $=0.9298$ and $\mathrm{Q}^{*}=0.8649$ (Figure-6).

The statistical calculi in the funnel plot were: Cochran- $\mathrm{Q}=12.03(\mathrm{P}<0.05), \mathrm{I}^{2}=83.4 \%$ and $\mathrm{T}^{2}=$ 0.9707. $\mathrm{P}<0.05$ suggested the presence of heterogeneity in the individual studies far from what would be expected singly. $\mathrm{I}^{2}>50 \%$ gave a dimension of the percentage of total variation of the studies due to heterogeneity (Figure-7).

\section{Analysis of SVI}

The same three articles that evaluated EPE also evaluated SVI with $1.5 \mathrm{~T}$ conventional images

Figure 5 - Florest plot of sensitivity and specificity for EPE.

A

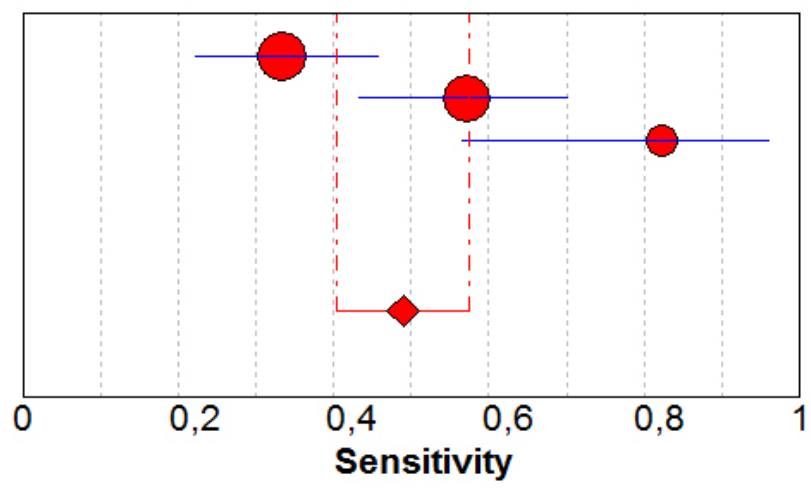

and showed a median sensitivity of 0.45 and specificity 0.96 .

The result of the homogeneity test for sensitivity was $\mathrm{Q}=9.98(\mathrm{P}=0.0068)$ and $\mathrm{I}^{2}=80.0 \%$, while for specificity $\mathrm{Q}=8.91(\mathrm{P}=0.0116)$ and $\mathrm{I}^{2}=77.6 \%$ (Figures 8a and 8b). sROC curve: AUC $=0.9241$ and $Q^{*}=0.8581$ (Figure-9).

Funnel plot obtained Cochran- $\mathrm{Q}=5.73(\mathrm{P}=$ $0.0570), \mathrm{I}^{2}=65.1 \%$ and $\mathrm{T}^{2}=1.1657 . \mathrm{P}=0.05$ and $\mathrm{I}^{2}$ lightly over $50 \%$ suggested the presence of discrete heterogeneity of the individual studies far from what expected singly. $\mathrm{T}^{2}>1$ suggested heterogeneity among the studies (Figure-10).

Funnel plot showed asymmetric format, suggesting the presence of bias publication (chance reasons away from the baseline vertical 1). Graphic with asymmetric tendency $\mathrm{P}=0.0570$ (limit of the significance statistical level), $\mathrm{I}^{2}>50 \%$ and $\mathrm{T}^{2}>1$ suggesting heterogeneity of the single studies, due to the variability of the size sample of each paper and due to the threshold effect for the diagnosis of SVI $(9,10)$.

$\begin{array}{lll}\text { Brajtbord 2011 } & 0,33 & (0,22-0,46) \\ \text { Janane 2011 } & 0,57 & (0,43-0,70) \\ \text { Kim 2010 } & 0,82 & (0,57-0,96)\end{array}$

Pooled Sensitivity $=0,49(0,40$ to 0,58$)$

Chi-square $=16,28 ; d f=2(p=0,0003)$

Inconsistency (I-square) $=87,7 \%$

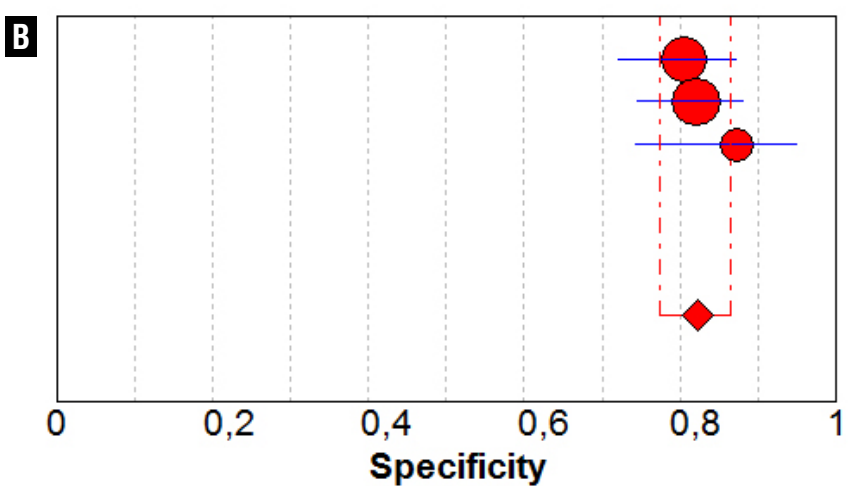

Specificity $(95 \% \mathrm{Cl})$

Brajtbord 2011

Janane 2011

Kim 2010

0,81

$(0,72-0,87)$

$0,82 \quad(0,75-0,88)$

$0,87 \quad(0,74-0,95)$

Pooled Specificity $=0,82(0,77$ to 0,86$)$

Chi-square $=1,09 ; \mathrm{df}=2(\mathrm{p}=0,5788)$

Inconsistency (I-square) $=0,0 \%$ 
Figure 6 - sROC curve depicting the representation of the sensitivity versus 1-specificity of EPE.

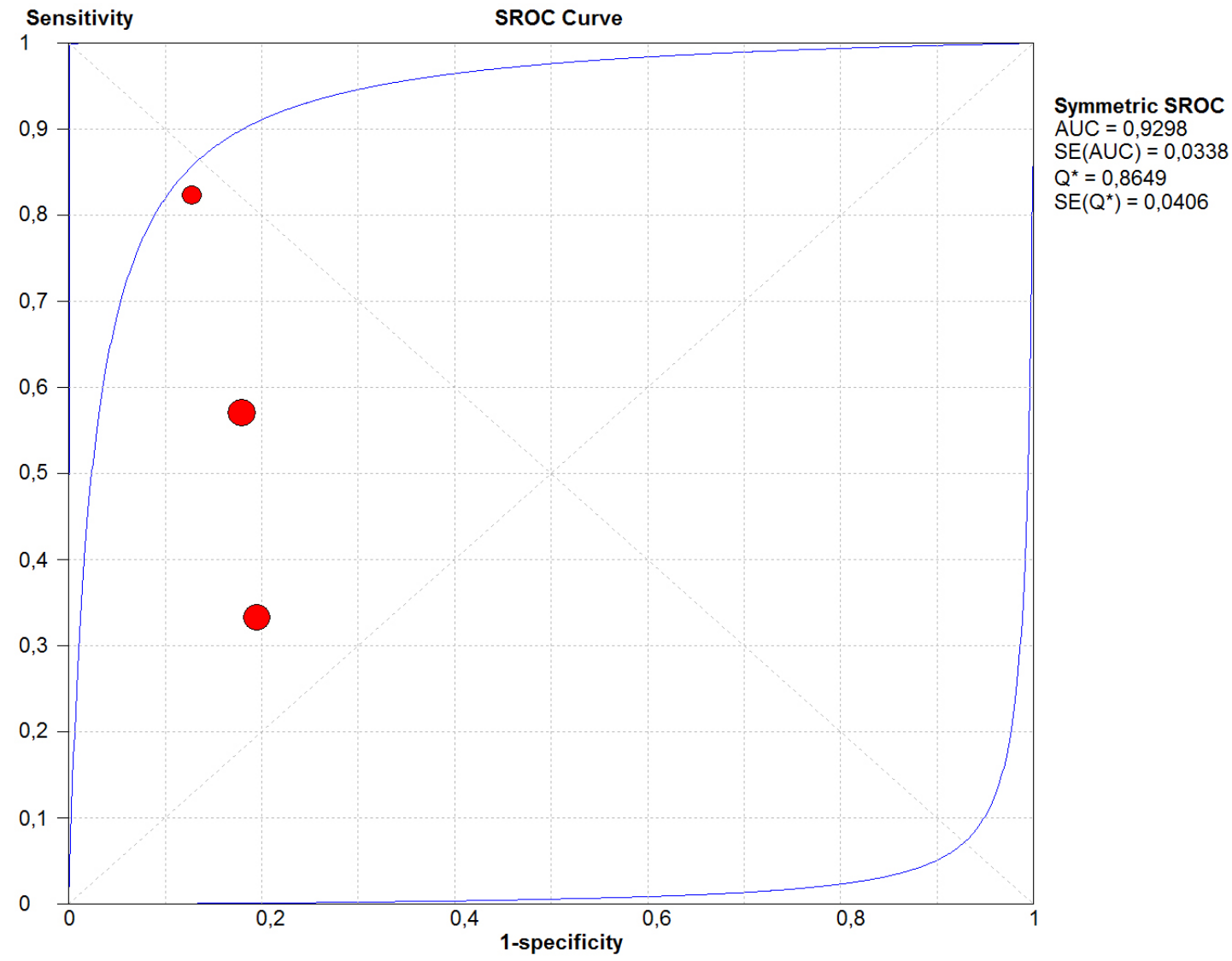

Figure 7 - Funnel plot of the studies regarding EPE.

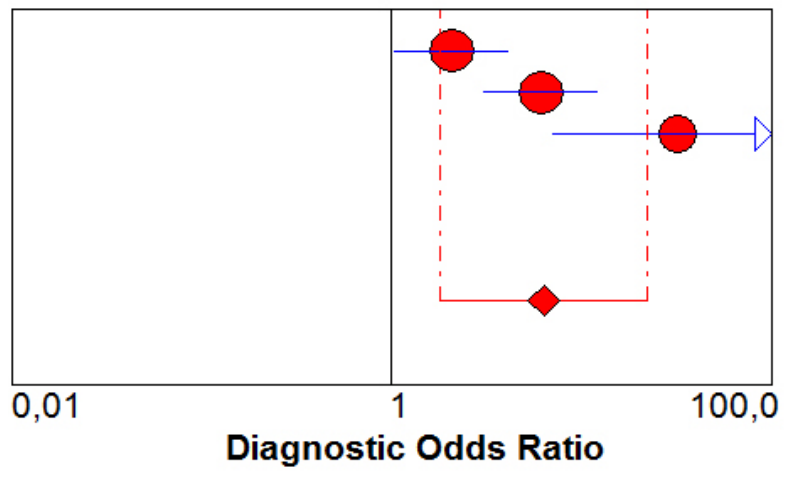

Diagnostic OR $(95 \% \mathrm{Cl})$

Brajtbord 2011

$2,07 \quad(1,04-4,13)$

$6,11 \quad(3,07-12,17)$

$31,89 \quad(7,02-144,77)$

Janane 2011

Kim 2010
Random Effects Model

Pooled Diagnostic Odds Ratio $=6,28(1,80$ to 21,87$)$

Cochran- $Q=12,03 ; d f=2(p=0,0024)$

Inconsistency (l-square) $=83,4 \%$

Tau-squared $=0,9707$ 
Figure 8 - Florest plot of sensitivity and specificity regarding VSI.

A

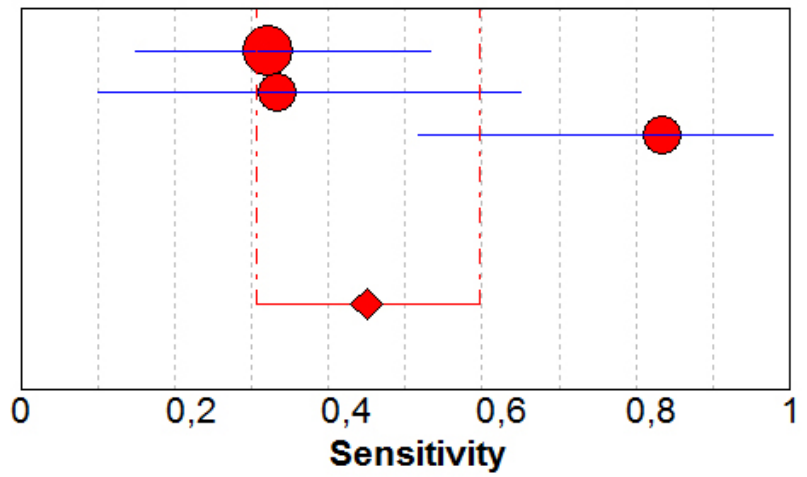

Brajtbord 2011

Janane 2011

Kim 2010

\section{Sensitivity $(95 \% \mathrm{Cl})$}

$0,32 \quad(0,15-0,54)$

$0,33 \quad(0,10-0,65)$

$0,83 \quad(0,52-0,98)$

Pooled Sensitivity $=0,45(0,31$ to 0,60$)$

Chi-square $=9,98 ; \mathrm{df}=2(\mathrm{p}=0,0068)$

Inconsistency (I-square) $=80,0 \%$

B

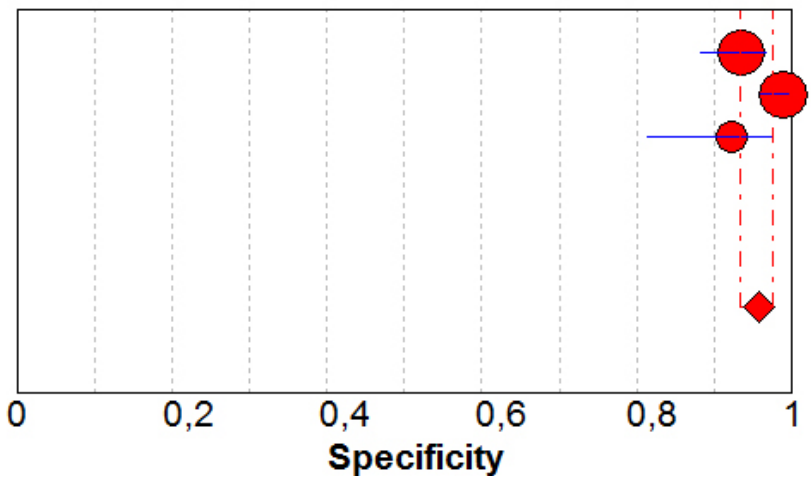

Specificity $(95 \% \mathrm{Cl})$

Brajtbord 2011

$0,93 \quad(0,88-0,97)$

$0,99 \quad(0,96-1,00)$

$0,92 \quad(0,81-0,98)$

\section{DISCUSSION}

In this SR all studies compared the histopathological results with pre-operatory MRI. Although there were more than 200 studies about MRI in the diagnosis and staging of PC, only seven were selected to evaluate pre-operatory $1.5 \mathrm{~T}$ MRI in patients submitted to RP compared to histopathologic results.

This limited number of studies must be analyzed carefully and will always evoke serious thoughts during systematic reviews, due to the small number of well elaborated articles, with a clear methodology and scientific relevance.

In relation to the selected articles, there were no calculi of the size of the sample in none of them. The studies were conducted in the respective institutions, without any common protocol, ignoring the differences and particularities of each study center. Only two of the seven studies
$(28.57 \%)$ were prospective and none of them informed financial disclosures. In spite of that, the analysis of quality of the studies using the QUADAS question form demonstrated that most questions regarding the study (more than 50\%) were answered "yes", suggesting that the quality of the studies was adequate.

Although independent, a few characteristics of the studies were the same in all, such as lack of previous treatment before PR, the use of 1.5T conventional images of MRI and a period of more than a month between the prostatic biopsy and RP surgery.

The difference of the values of sensitivity and specificity for the diagnosis of PC, as well as of the respective low medians, may be explained by the threshold limits or the cutoff of the particular exam.

Jeong et al. and Yoshizako et al. described the standardization of the diagnosis of PC in five 
Figure 9 - SROC curve depicting the representation of the sensitivity versus 1-specificitity of VSI.

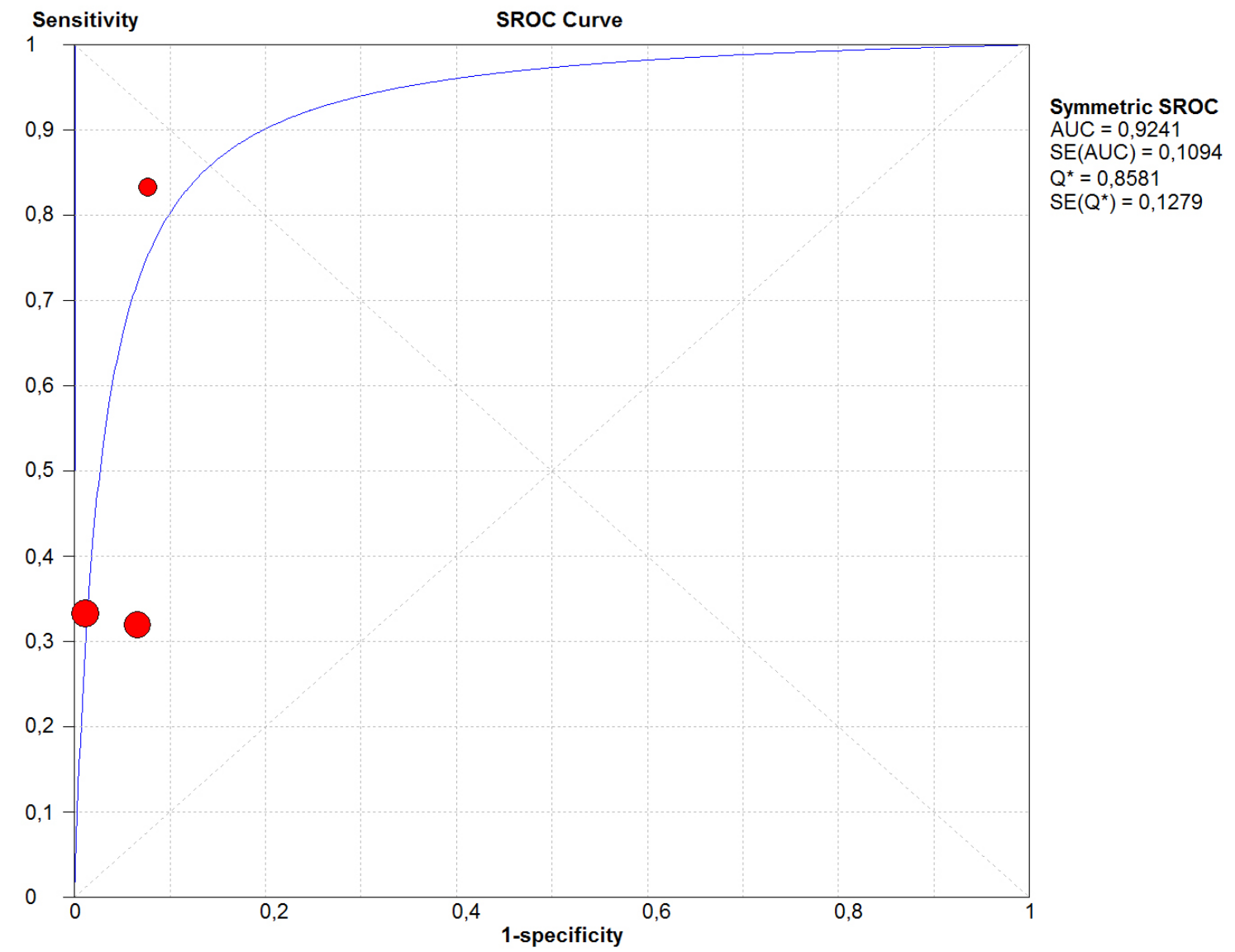

Figure 10 - Funnel plot of the studies regarding VSI.

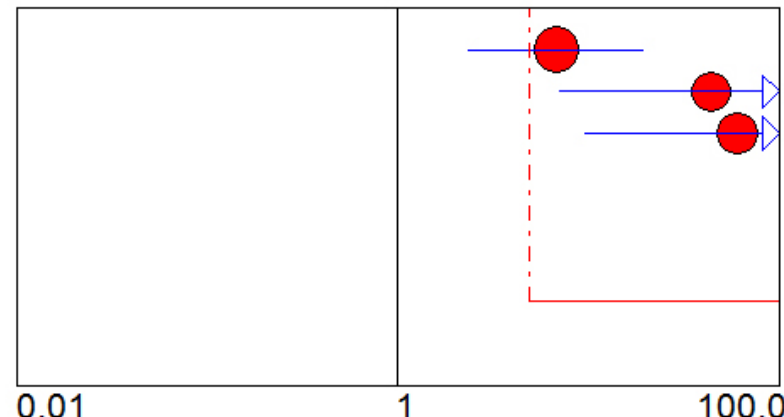

Diagnostic Odds Ratio
Diagnostic OR $(95 \% \mathrm{Cl})$

Brajtbord 2011

Janane 2011

$6,73 \quad(2,34-19,36)$

$44,00 \quad(6,99-276,86)$

$60,00 \quad(9,63-373,66)$

Random Effects Model

Pooled Diagnostic Odds Ratio $=22,24(4,87$ to 101,52$)$

Cochran- $Q=5,73 ; d f=2(p=0,0570)$

Inconsistency $($ I-square $)=65,1 \%$

Tau-squared $=1,1657$ 
categories: cancer certainly absent, cancer probably absent, cancer possibly present, cancer probably present and cancer certainly present $(12,13)$. Other authors categorized in two options: presence or absence of cancer. Also, in only those two studies it was clear that the radiological evaluation was made by two well trained radiologists. Another aspect is the lack of standardization of the radiological parameters for the diagnosis of PC used by most studies, and some actually don't mention them. Finally, the size of the tumor was not considered for the diagnosis of PC, except on the study of Janane et al. (14).

Chabanova et al. studied conventional images of MRI and spectroscopy and perfusion methods in the diagnosis of PC. In relation to spectroscopy, they found sensitivity of 0.46 and specificity of 0.78 . In relation to perfusion, sensitivity and specificity were respectively 0.48 and 0.68 . They demonstrated that the combination of conventional MRI, spectroscopy and perfusion could diagnose all patients with PC, indicating a positive combination of the three methods. However, this association is expensive and troublesome, and can derail the routine use of MRI (15).

Yoshizako et al. also studdied the combination of conventional MRI images with difusion and perfusion. The combination of conventional MRI and diffusion showed sensitivity of 0.80 and specificity of 0.87 , and when combined with perfusion showed sensitivity of 0.69 and specificity of 0.68 . The combination of the three methods showed sensitivity of 0.69 and specificity of 0.93 . The combination of the three methods was more accurate for the diagnosis of PC than conventional MRI alone, but this data referred only to PC of the prostatic transition zone (13).

Jeong et al. studied the conventional images and the combination of them with the diffusion method and found sensitivity and specificity of 0.87 and 0.72 respectively. The authors did not report the data of the diffusion method alone (12).

Beyersdorff et al. compared conventional MRI with perfusion images alone. In relation to perfusion, the sensitivity and specificity were 0.78 and 0.79 respectively. The sensitivity was superior to that of conventional MRI $(0.60)$ and the specificity a little lower than that of conventional MRI
(0.82) (16). The heterogeneity of the studies can be explained by the variable size of the sample of each study, by the different criteria to diagnose PC using MRI and by the different studied populations of each study (8).

The suspicion of EPE at MRI is made by specific signs, as the presence of solid tissue in the periprostatic fat, irregular bulging of the prostatic capsule and obliteration of the retoprostatic angle, as well as non-specific signs as capsular thickening, capsular retraction and regular bulging of the capsule (17).

The values of sensitivity of the three studies that evaluated EPE are different, leading to a considerable heterogeneity of the individual studies $\left(\mathrm{P}<0.05\right.$ and $\mathrm{I}^{2}$ greater than $\left.50 \%\right)$ and medium of 0.49 (CI 95\%: $0.40-0.58$ ).

Kim et al. considered EPE the presence of obliteration of the retoprostatic angle, blurring of the periprostatic fat, invasion of the neurovascular bundle or transcapsular tumor continuation (18). Janane et al also included discontinuity of the prostatic capsule, obliteration of the prostatic veins and a distance between the tumor and the prostatic capsule equal or superior to $1 \mathrm{~cm} \mathrm{(14).}$ Brajtbord et al didn't disclosure the used criteria to consider the presence of EPE as a positive MRI (19).

Another important aspect was that the analyzed populations were different among the revised studies. Janane et al included a particular north-african population and didn't mention the categorization of the population according to extra-prostatic risk of the disease (14). Kim et al excluded low risk patients and included only high or intermediate risk patients (18). Brajtbord et al didn't state the categorization risk, although presented the histopathological results: Gleason $<7$ : 13\%, Gleason = 7: 69\%, Gleason > 7: 16\% (19).

The reduced sensitivity rates for EPE are due to the incapacity of the used images techniques, including MRI, to detect microscopic extra-prostatic tumors. The low specificity is due to the difficulty of the image techniques to distinguish prostatic tumors and benign inflammatory diseases (20).

Regarding the study of SVI sensitivity, there was a discrepancy between Kim study and 
the two others, with heterogeneity $\left(\mathrm{P}<0.05\right.$ and $\mathrm{I}^{2}>$ $50 \%$ ). Kim et al worked exclusively with intermediate and high risk patients, increasing the MRI sensitivity in relation to the two other authors (18).

When evaluating the specificity, each individual study presented results greater than 0.9, with a median of 0.96 (IC 95\%: 0.93 - 0.98). Kim et al suggested the presence of VSI when there was a low intensity sign causing dilation and an asymmetric gland (18). Janane et al identified the VSI as the focal shortening of the wall or the presence of low intensity sign inside the vesicles (14). Brajtford et al only informed presence or absence of VSI (17).

In this SR, it is concluded that MRI with 1.5T conventional images using endorectal coil should not be routinely used in all patients with PC. It should only be used as a complementary method for the diagnosis and staging of the disease and indicated for only specific patients.

More studies evaluating new technologies (for example, 3T MRI) and multiple parameters (diffusion, perfusion and spectroscopy) are necessary before recommending the routine use of MIR in clinical practice.

\section{CONCLUSIONS}

There are very few good studies comparing pre-operatory pelvic MRI using $1.5 \mathrm{~T}$ conventional images and histopathological results of patients with PC submitted to RP. Also, the analysed studies showed a significant heterogeneity. The best results of SR are related to specificity for VSI. This SR suggests that 1.5T MRI using endorectal coil is not indicated routinely for the diagnosis and staging of patients with PC.

\section{CONFLICT OF INTEREST}

None declared.

\section{REFERENCES}

1. Hsing AW, Chokkalingam AP: Prostate cancer epidemiology. Front Biosci. 2006; 11: 1388-413.
2. Chodak GW: The role of watchful waiting in the management of localized prostate cancer. J Urol. 1994; 152: 1766-8.

3. Dodds PR, Caride VJ, Lytton B: The role of vertebral veins in the dissemination of prostatic carcinoma. J Urol. 1981; 126: 753-5.

4. Lorente JA, Morote J, Raventos C, Encabo G, Valenzuela $\mathrm{H}$ : Clinical efficacy of bone alkaline phosphatase and prostate specific antigen in the diagnosis of bone metastasis in prostate cancer. J Urol. 1996; 155: 1348-51.

5. Abuzallouf S, Dayes I, Lukka H: Baseline staging of newly diagnosed prostate cancer: a summary of the literature. J Urol. 2004; 171: 2122-7.

6. lared W, Valente 0: Revisões sistemáticas de estudos de acurácia. Diagn Tratamento. 2009; 14: 85-8.

7. Moher D, Cook DJ, Eastwood S, Olkin I, Rennie D, Stroup DF: Improving the quality of reports of meta-analyses of randomised controlled trials: the QUOROM statement. Quality of Reporting of Meta-analyses. Lancet. 1999; 354: 1896-900.

8. Higgins JP, Thompson SG, Deeks JJ, Altman DG: Measuring inconsistency in meta-analyses. BMJ. 2003; 327: 557-60.

9. Walter SD: Properties of the summary receiver operating characteristic (SROC) curve for diagnostic test data. Stat Med. 2002; 21: 1237-56.

10. Egger M, Smith GD: Bias in location and selection of studies. BMJ. 1998; 316: 61-6.

11. Moayyedi P: Meta-analysis: Can we mix apples and oranges? Am J Gastroenterol. 2004; 99: 2297-301.

12. Jeong IG, Kim JK, Cho KS, You D, Song C, Hong JH, et al.: Diffusion-weighted magnetic resonance imaging in patients with unilateral prostate cancer on extended prostate biopsy: predictive accuracy of laterality and implications for hemi-ablative therapy. J Urol. 2010, 184: 1963-9.

13. Yoshizako T, Wada A, Hayashi T, Uchida K, Sumura M, Uchida $\mathrm{N}$, et al.: Usefulness of diffusion-weighted imaging and dynamic contrast-enhanced magnetic resonance imaging in the diagnosis of prostate transition-zone cancer. Acta Radiol. 2008; 49: 1207-13.

14. Janane A, Hajji F, Ismail TO, Elondo JC, Ghadouane M, Ameur A, et al.: WITHDRAWN: Endorectal MRI accuracy in auguring tumour location, tumour extent, capsular perforation and seminal vesicle invasion of prostate cancer in north-African men. Eur J Radiol. 2011; 26. [Epub ahead of print]

15. Chabanova E, Balslev I, Logager V, Hansen A, Jakobsen H, Kromann-Andersen B, et al.: Prostate cancer: $1.5 T$ endocoil dynamic contrast-enhanced MRI and MR spectroscopy--correlation with prostate biopsy and prostatectomy histopathological data. Eur J Radiol. 2011; 80: 292-6.

16. Beyersdorff $D$, Lüdemann L, Dietz E, Galler D, Marchot $P$, Franiel T. Dynamic contrast-enhanced MRI of the prostate: comparison of two different post-processing algorithms. Rofo. 2011; 183: 456-61. 
17. Yu KK, Hricak H, Alagappan R, Chernoff DM, Bacchetti P, Zaloudek CJ: Detection of extracapsular extension of prostate carcinoma with endorectal and phased-array coil MR imaging: multivariate feature analysis. Radiology. 1997; 202: 697-702.

18. Kim B, Breau RH, Papadatos D, Fergusson D, Doucette S, Cagiannos I, et al.: Diagnostic accuracy of surface coil magnetic resonance imaging at $1.5 \mathrm{~T}$ for local staging of elevated risk prostate cancer. Can Urol Assoc J. 2010; 4: 257-62.
19. Brajtbord JS, Lavery HJ, Nabizada-Pace F, Senaratne P, Samadi DB: Endorectal magnetic resonance imaging has limited clinical ability to preoperatively predict $\mathrm{pT} 3$ prostate cancer. BJU Int. 2011; 107: 1419-24.

20. Epstein JI, Chan DW, Sokoll LJ, Walsh PC, Cox JL, Rittenhouse $\mathrm{H}$, et al.: Nonpalpable stage T1c prostate cancer: prediction of insignificant disease using free/total prostate specific antigen levels and needle biopsy findings. J Urol. 1998; 160: 2407-11.

Correspondence address: Dr. Rogério Cardoso da Silva Department of Uro-oncology, Universidade Estadual de Campinas, UNICAMP

Campus Universitario Zeferino Vaz SN Cidade Universitaria - Campinas, 13083-970 E-mail: rogeriocardosodasilva@hotmail.com 